\title{
Dirac particles in Rindler space
}

\author{
Michael Soffel, Berndt Müller, and Walter Greiner \\ Institut für Theoretische Physik, Johann Wolfgang Goethe Universität, D6000 Frankfurt/Main, Germany \\ (Received 6 June 1979)
}

We show that a uniformly accelerated observer experiences a "thermal" flux of Dirac particles in the ordinary Minkowski vacuum.

It has been known for several years ${ }^{1-3}$ that the particle interpretation of quantum field theory in a general Riemannian manifold is not unique, but depends on the observer's coordinate frame. The particle concept, endowed with a nonlocal nature by the uncertainty relation, depends on the global topological structure of that submanifold which is naturally connected with the observer's state of motion.

In this article we show that a uniformly accelerated observer in Minkowski space $\mathfrak{i}$ experiences a flux of Dirac particles in the ordinary Minkowski vacuum. To do this we utilize a method of Rumpf, ${ }^{4-7}$ allowing one to define particle and antiparticle states in quite general circumstances.

Owing to the dynamics of the uniformly accelerated obse rver, two-dimensional Minkowski space is divided into four sectors, as can be seen from Fig. 1: right (I), left (II), future $(F)$, and past $(P)$ with respect to the origin $x=t=0$. Minkowski coordinates $(t, x)$ may be transformed into Rindler coordinates $(v, u)$ according to [the following conventions are used: $\hbar=c=1,\left(x^{0}=t, x^{3}=x\right)$ in $\mathfrak{N}$, $\left(x^{0}=v, x^{3}=u\right)$ in I $\cup$ II, and $\left(x^{0}=u, x^{3}=v\right)$ in $\left.F \cup P\right]$

$t=u \sinh v, \quad x=u \cosh v$,

in I, II (1a)

$v=\operatorname{arctanh}(t / x), \quad u=\operatorname{sgn} x\left(x^{2}-t^{2}\right)^{1 / 2}$,

$t=u \cosh v, \quad x=u \sinh v$,

$v=\operatorname{arctanh}(x / t), u=\operatorname{sgn} t\left(t^{2}-x^{2}\right)^{1 / 2}$.

This leads to the line element

$$
\begin{aligned}
& d s^{2}=u^{2} d v^{2}-d u^{2} \text { in I, II , } \\
& d s^{2}=d u^{2}-u^{2} d v^{2} \text { in } F, P .
\end{aligned}
$$

It is well known that a world line $u=$ const in I corresponds to the trajectory of a uniformly accelerated observer. In I the timelike coordinate $v$ is connected with the observer's proper time via $v=g \tau$ where $g$ is the observer's acceleration. We start calculating Dirac wave functions in the four sectors of Minkowski space. The covariant Dirac equation reads ${ }^{8}$

$$
\left[i \gamma^{\mu}\left(\partial_{\mu}+\Gamma_{\mu}\right)-m\right] \psi=0 .
$$

Let us first concentrate on sector I. $\partial_{v}$ being a (timelike) Killing vector, we may solve for stationary states $u(v, u)=\psi(u) \exp (-i \omega v)$ :

$$
\omega \psi=\left[-i \alpha_{3} u^{1 / 2} \frac{\partial}{\partial u} u^{1 / 2}+\beta m u\right] \psi \equiv \hat{H}_{R} \psi
$$

For spin-up states the only normalizable solution reads $^{9}$

$$
\psi(u)=\left[\begin{array}{c}
\phi_{\omega}^{-}+\phi_{\omega}^{+} \\
0 \\
\phi_{\bar{\omega}}^{-}-\phi_{\omega}^{+} \\
0
\end{array}\right], \quad \phi_{\omega}^{ \pm}=H_{i \omega \pm 1 / 2}^{(1)}(i m u)
$$

with $\phi_{\omega}^{ \pm}$obeying the differential equation

$$
\left(u \frac{d}{d u} u \frac{d}{d u}\right) \phi_{\omega}^{ \pm}=\left[m^{2} u^{2}-\left(\omega \mp \frac{i}{2}\right)^{2}\right] \phi_{\omega}^{ \pm} \quad(\omega \in \mathrm{R}) \text {. }
$$

It is interesting to note that the difference in the boson case is the term $\frac{1}{2} i$ in Eq. (6) which does not

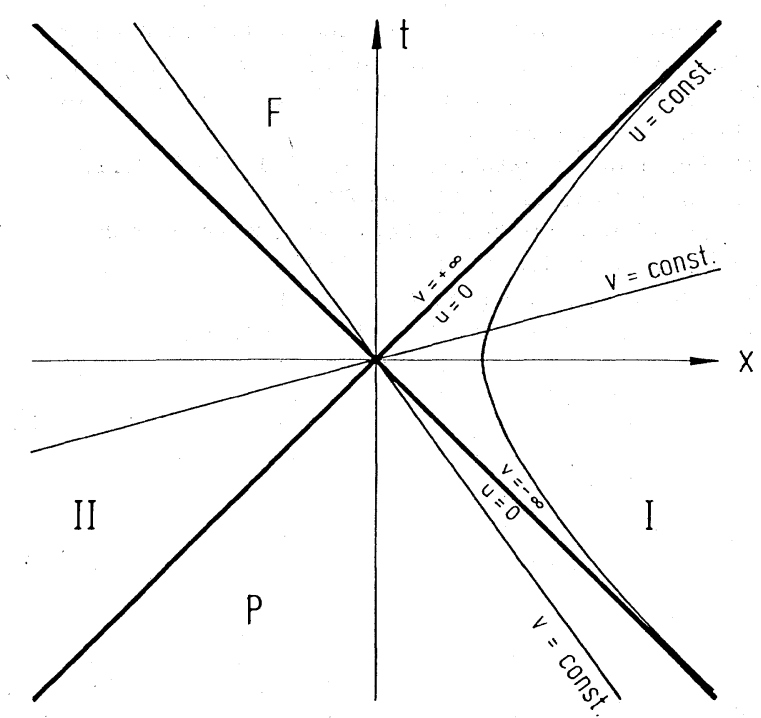

FIG. 1. Owing to the dynamics of the uniformly accelerated observer, two-dimensional Minkowski space is divided into four parts: right (I), left (II), future $(F)$, and past $(P)$. 
appear in the Klein-Gordon Equation. This term arises due to the nonvanishing derivative of $\hat{H}_{R}$ [Eq. (4)]. Let us denote the solution of the Dirac equation in sector I as

$$
\mathrm{I}_{\omega}=e^{-i \omega v}\left[\begin{array}{c}
\phi_{\omega}^{-}+\phi_{\omega}^{+} \\
0 \\
\phi_{\bar{\omega}}^{-}-\phi_{\omega}^{+} \\
0
\end{array}\right] \theta(t+x) \theta(x-t)
$$

indicating that $I \subset \mathfrak{M}$ is the support of ${ }^{\mathrm{I}} \mho_{\omega}$. The norm of ${ }^{\mathrm{I}} \psi_{\omega}$ is given by

$$
\left\langle{ }^{\mathrm{I}} \psi_{\omega},{ }^{\mathbf{I}} \psi_{\omega^{\prime}}\right\rangle=\frac{8 e^{\pi \omega}}{m \cosh (\pi \omega)} \delta\left(\omega-\omega^{\prime}\right),
$$

where the Dirac scalar product $\langle$,$\rangle is defined as$

$$
\left\langle\psi_{1}, \downarrow_{2}\right\rangle=\int_{\Sigma} d \sigma_{\mu} \psi_{1}^{\dagger} \beta \gamma^{\mu} \psi_{2}
$$

on some spacelike hypersurface $\Sigma$. In the future sector $(F), u$ and $v$ interchange their meaning as spacelike or timelike coordinates. Equation (3) therefore takes the form

$$
\omega \psi=\left(-i \alpha_{3} u^{1 / 2} \frac{\partial}{\partial u} u^{1 / 2}+\tilde{\gamma}_{3} m u\right) \psi
$$

where $\tilde{\gamma}_{3}$ is the Dirac matrix in Minkowski space. We obtain the following two independent solutions for Eq. (10):

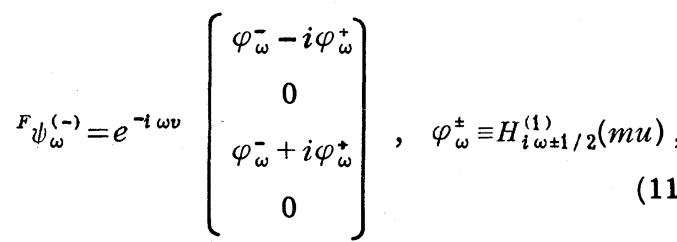

$$
\begin{aligned}
& F_{\omega}^{(+)}=e^{-i \omega v}\left[\begin{array}{c}
\tilde{\varphi}_{\omega}^{-}-i \tilde{\varphi}_{\omega}^{+} \\
0 \\
\tilde{\varphi}_{\omega}^{-}+i \tilde{\varphi}_{\omega}^{+} \\
0
\end{array}\right], \quad \tilde{\varphi}_{\omega}^{ \pm} \equiv H_{i \omega \pm 1 / 2}^{(2)}(m u)
\end{aligned}
$$

with

$$
\left\langle{ }^{F} \psi_{\omega}^{(i)}, F^{F} \psi_{\omega^{\prime}}^{(k)}\right\rangle=\frac{16 e^{-\pi \omega k}}{m} \delta\left(\omega-\omega^{\prime}\right) \delta_{i k}(i, k= \pm) .
$$

We obtain analogous wave functions in II and $P$ through

$$
\begin{aligned}
& { }^{\mathrm{II}} \psi_{\omega}(t, x)={ }^{\mathrm{I}} \psi_{\omega}(-t,-x), \\
& { }^{P} \psi_{\omega}^{( \pm)}(t, x)={ }^{F} \psi_{\omega}^{( \pm)}(-t,-x) .
\end{aligned}
$$

We may now obtain the usual quantum field theory in Minkowski space by constructing an ortho- normal basis of particle (antiparticle) wave functions $\psi_{\omega}^{( \pm)}$of the homogeneous Dirac equation in all $\mathscr{M}$ by joining ${ }^{\mathrm{I}} \mathrm{II}_{\mho_{\omega}}$ and ${ }^{F, P} \psi_{\omega}^{(t)}$. To this end we use Rumpf's definition of particle and antiparticle modes: A solution of the Dirac equation is an outgoing/ingoing particle (antiparticle) mode if it admits an analytical continuation in $m$ such that it remains regular, except in the past/future, if $m^{2}$ acquires a negative/positive (positive/negative) imaginary part.

To apply this definition, we use the asymptotic representation of $\varphi_{\omega}^{ \pm}$for large times $\mathrm{u}$ (in sector $F)$ :

$$
\varphi_{\omega}^{ \pm} \underset{u \rightarrow \infty}{\sim}\left(\frac{2}{\pi m u}\right)^{1 / 2} \exp \left[i m u-i\left(\frac{\pi}{4}+\frac{\pi}{2}\left(i \omega \pm \frac{1}{2}\right)\right)\right] .
$$

From the properties of the complex exponential function it is obvious that ${ }^{F} \psi_{\omega}^{(+)}\left({ }^{F} \psi_{\omega}^{(-)}\right)$must be classified as part of a particle (antiparticle) mode. The mode classification in $P$ is obtained from Eq. (13). Thus, by Rumpf's definition, $\psi_{\omega}^{ \pm}$may be expressed as

$$
\begin{aligned}
& \psi_{\omega}^{+}=N\left(a_{1}{ }^{\mathrm{I}} \psi_{\omega}+a_{2}{ }^{F_{\psi}} \psi_{\omega}^{(+)}+a_{3}{ }^{P} \psi_{\omega}^{(-)}+a_{4}{ }^{\mathrm{II}} \psi\right), \\
& \psi_{\omega}^{-}=M\left(b_{1}{ }^{\mathrm{I}} \psi_{\omega}+b_{2}{ }^{F} \psi_{\omega}^{(-)}+b_{3}{ }^{P} \psi_{\omega}^{(+)}+b_{4}{ }^{\mathrm{II}} \psi\right),
\end{aligned}
$$

where the constants $a_{i}$ and $b_{i}$ have to be determined by the requirement that $\psi_{\omega}^{ \pm}$should obey the homogeneous wave equation in $\Re$.

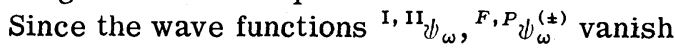
outside of their support they are not solutions of Dirac's Eq. (3) in $\mathfrak{M}$ but possess distributions as source terms on the light cone through the origin of $\mathfrak{M}$. By calculating the source terms for the various wave functions the following complete, orthonormal set of wave functions is obtained:

$$
\begin{aligned}
\psi_{\omega}^{+}=\left(\frac{m}{48}\right)^{1 / 2} & \left({ }^{\mathrm{I}} \psi_{\omega}-e^{\pi \omega / 2 F} \psi_{\omega}^{(+)}\right. \\
& \left.+e^{-\pi \omega / 2} P_{\psi_{\omega}^{(-)}}-i e^{-\pi \omega \mathrm{II}} \psi_{\omega}\right), \\
\psi_{\omega}^{-}=\left(\frac{m}{48}\right)^{1 / 2} & \left(e^{-\pi \omega \mathrm{I}} \psi_{\omega}+e^{-\pi \omega / 2 F} \psi^{(-)}\right. \\
& \left.+e^{\pi \omega / 2 P_{\psi}} \psi_{\omega}^{(+)}+i^{\mathrm{II}} \psi_{\omega}\right) .
\end{aligned}
$$

The usual quantum field theory on $\mathfrak{T}$ is obtained by means of the following spectral representation of the Dirac operator $\hat{\psi}$ :

$$
\begin{aligned}
& \hat{\psi}=\sum_{s} \int_{-\infty}^{+\infty} d \omega\left(\hat{a}_{\omega, s} \psi_{\omega, s}^{+}+\hat{b}_{\omega, s}^{\dagger} \psi \bar{\omega}, s\right), \\
& \hat{a}_{\omega}\left|0_{M}\right\rangle=\hat{b}_{\omega}\left|0_{M}\right\rangle=0
\end{aligned}
$$

( $s$ now sums over spin states).

Suppose a uniformly accelerated observer moving on a $u=$ const trajectory in ICM (Fig. 1). Unruh and Rumpf ${ }^{3,4}$ have shown that he measures only wave functions on sectors which are causally connected with himself. His world is therefore de- 
scribed by the unique normalized wave functions of the homogeneous equation in $B=I \cup F \cup P$ :

$$
\begin{aligned}
\psi_{\omega}=\frac{m^{1 / 2} e^{-\pi \omega / 2}}{[96 \cosh (\pi \omega)]^{1 / 2}} & 2 \cosh (\pi \omega)^{\mathrm{I}} \psi_{\omega}+e^{-\pi \omega / 2 F} \psi_{\omega}^{(-)} \\
& -e^{3 \pi \omega / 2 F_{\psi_{\omega}^{(+)}}+e^{\pi \omega / 2 P} \psi_{\omega}^{(-)}} \\
& \left.+e^{\pi \omega / 2 P} \psi_{\omega}^{(+)}\right]
\end{aligned}
$$

Note that $\psi_{\omega}$ does not contain ${ }^{\mathrm{II}} \psi_{\omega}$ because the observer's information is decoupled from $\Pi \subset \mathfrak{M}$. It is at this point where the global aspect of the submanifold $B$ plays a crucial role for the particle interpretation of $\hat{\psi}$.

The observer modes are related to the Minkowski modes according to

$$
\psi_{\omega}=\alpha_{\omega} \psi_{\omega}^{+}+\beta_{\omega} \psi_{\omega}^{-}
$$

with

$$
\alpha_{\omega}=\frac{e^{\pi \omega / 2}}{[2 \cosh (\pi \omega)]^{1 / 2}}, \quad \beta_{\omega}=\frac{e^{-\pi \omega / 2}}{[2 \cosh (\pi \omega)]^{1 / 2}} .
$$

Now Unruh ${ }^{3}$ and others have shown that the accelerated observer experiences a spectral representation of $\hat{\psi}$ according to positive and negative values of $\omega$ :

$$
\hat{\psi}^{B}=\sum_{s} \int_{0}^{\infty} d \omega\left(\hat{\boldsymbol{c}}_{\omega, s^{\psi}{ }_{\omega, s}}+\hat{d}_{\omega, s}^{\dagger} \psi_{-\omega, s}\right),
$$

defining the $B$ vacuum

$$
\hat{c}_{\omega}\left|0_{B}\right\rangle=\hat{d}_{\omega}\left|0_{B}\right\rangle=0 \text {. }
$$

We may calculate the number of $B$ particles in the ordinary Minkowski vacuum as $\left(\omega, \omega^{\prime}>0\right)$

$$
\left\langle 0_{M}\left|\hat{\boldsymbol{c}}_{\omega, s}^{\dagger} \hat{\boldsymbol{c}}_{\omega^{\prime}, s^{\prime}}\right| 0_{M}\right\rangle=\beta_{\omega^{2}}{ }^{2} \delta\left(\omega-\omega^{\prime}\right) \delta_{s s^{\prime}} .
$$

This can be interpreted that the observer measures a number of created particles per unit interval of proper time:

$$
\frac{d n}{d \tau}=2 \int_{0}^{\infty} \frac{d \omega / 2 \pi}{e^{2 \pi \omega / g}+1}
$$

where the factor of 2 is introduced by the two spin projections. This means that he measures a "thermal" flux of Dirac particles where the effective "temperature" is the Fulling-Unruh temperature

$$
T_{B}=\frac{g \hbar}{2 \pi c k}=10^{-23} \mathrm{~g}^{\circ} \mathrm{K} \mathrm{sec}^{2} / \mathrm{cm} .
$$

Thus one expects that the action of the Dirac field onto a particle detector corresponds to the action of an isotropic temperature bath of temperature $T_{B}$. Note that the change from the boson to fermion statistics resulted from the details of the Dirac equation, i.e., the additional term $\frac{1}{2} i$ in the Dirac equation changes the behavior of the wave functions at $u=0$ as compared to the Klein-Gordon equation. This leads to $\beta_{\omega}=\left(e^{2 \pi \omega}+1\right)^{-1 / 2}$ instead of $\beta_{\omega}=\left(e^{2 \pi \omega}-1\right)^{-1 / 2}$ as was found by Fulling ${ }^{1}$ and Rumpf ${ }^{4}$ for scalar particles.

We finally note that a pure quantum state defined on the whole Minkowski manifold may appear as a mixed state when measured on a submanifold for the following reason: Since the observer modes $\psi_{\omega}$ vanish on sector II $\subset \mathfrak{M}, \hat{\psi}^{B}$ generates only a subalgebra of the whole Minkowski field algebra. Let $\hat{P}_{B}$ be the projector onto the set of all states generated by $\left|0_{B}\right\rangle$. Then $\hat{P}_{B}\left|0_{M}\right\rangle$ will be a mixed state containing particle antiparticle excitations.

This work was supported by the Deutsche Forschungsgemeinschaft (DFG).
${ }^{1}$ S. Fulling, Phys. Rev. D $\underline{7}, 2850$ (1973).

${ }^{2}$ P. C. Davies, J. Phys. A $\overline{8}, 609$ (1975).

${ }^{3}$ W. Unruh, Phys. Rev. D $\overline{14}, 870$ (1976).

${ }^{4} \mathrm{H}$. Rumpf, dissertation, Wien, 1977 (unpublished).

${ }^{5} \mathrm{H}$. Rumpf, Phys. Lett. 61B, 272 (1976).

${ }^{6}$ H. Rumpf, Nuovo Cimento 35B, 321 (1976).
${ }^{7}$ H. Rumpf, Gen. Relativ. Gravit. (to be published).

${ }^{8}$ M. Soffel, B. Müller, and W. Greiner, J. Phys. A 10 , 551 (1977).

${ }^{9}$ M. Abramowitz and I. Stegun, Handbook of Mathematical Functions (Dover, New York, 19́70). 